\title{
MATERIALS NEEDED FOR THE LIBRARY
}

The Rutgers University Library lacks files of the following periodicals. If any friends can furnish them, the Library will be grateful:

\section{Agriculture}

Agricultural Engineering, Vols. I-IO (1920-1929).

Agricultural History. All volumes.

Arborist's News. Vol. 2 (1936-to date).

Horticulture. Vols. I-4 (1923-1926).

\section{Engineering}

Institution of Chemical Engineers-Transactions. London. v. 4 (1926)

Institution of Electrical Engineers-Journal. Lond. v. 34-35 (1904-05) v. $37-39$ (Ig06-8), v. 4I-42 (I 908-9), v. 45-47 (I IIO-I2), v. 60 (I921-2)

Institution of Mechanical Engineers-Proceedings. England. 1847, 1909-22

Municipal Sanitation. N. Y. v. I (1930)

National Electric Light Association-Bulletin. N. Y. v. I-6 (I907-July I9I3) ns. v. I-I I (Aug. I9I3-1924)

Proceedings. N. Y. 3 Ist v. 2 (1908), 47th (1924), 5oth-56th (1927-1933)

New England Water Works Association-Journal. Boston, Mass. v. I-9 (I 886-94), v. 22-25 (I908-I I)

\section{Science}

American Journal of Anatomy. Phila. v. I2 (I9I I I I2)

Annals of Applied Biology. London. v. 2 (IgI5)

Biochemical Journal. London. v. 4-6 (I $909^{-1}$ I ), v. 9-I I (I I I $^{-1}$ 7)

Bulletin of Entomological Research. v. I 8-20 (1927-29) London.

Chemical and Metallurgical Engineering. N. Y. v. 3 (1905)

Faraday Society-Transactions. v. I-2 (Ig06-7), v. I2-I9 (IgI6-24), v. 2I22 (1925-27) London.

Entomological Society of America-Annals. Columbus, O. v. 9-10 (I $\left.9^{16-17}\right)$

Franklin Institute Journal. Phila. v. 5 (Jan.-June 1828), v. 9 (Jan.-June I830), v. I3 (Jan.-June I832), v. I7 (Jan.-June I834), v. I9-2I (Jan. I 835-June 1836), v. 23-76 (Jan. I837-Dec. I863), v. 79 (Jan.-June I 865), v. 81-IO2 (Jan. I866-Dec. I876), v. I08 (July-Dec. I879) v. I I0I 2 (Jl. I 880-Dec. I88I), v. II4 (Jl.-Dec. I882), v. I I6 (Jl.-Dec. I883)

Isis. Brussels, Belgium. v. I (IgI3). 